\title{
Electromyographic power spectral analysis of jaw opening and closing muscles during fatigue
}

\author{
Yoshiaki Yamada, Hiroyuki Suzuki*, Hisao Murakami and Michinobu Irie* \\ Department of Physiology (Chief: Prof. Toshihide Sato) and \\ *Department of Orthodontics (Chief: Prof. Michinobu Irie), \\ School of Dentistry, Nagasaki University, \\ 7-1 Sakamoto, Nagasaki 852, Japan
}

[Accepted 'for publication:

Key words: muscular fatigue / EMG / power spectrum / jaw muscles

\section{Introduction}

Electromyography (EMG) has been used for both basic and clinical studies. The EMG contains more information than just the degree of muscle contraction. Much research has been done on the influence of local muscular fatigue on the signal characteristics of the EMG. One of the most prominent changes in the EMG during an isometric fatiguing contraction is the shift of the EMG power spectrum to lower frequencies.

Functional disturbances of the masticatory system usually cause muscular pain. The pain seems to be mainly due to muscular fatigue. In fact, forced biting results in muscular fatigue accompanied by pain ${ }^{1,2)}$. Therefore, power spectral analysis was carried out on the masticatory muscles ${ }^{3-5}$.

Since surface EMG is composed of many muscle action potentials, several mechanisms are proposed; 1) The duration of the action potentials from individual muscle fiber may become longer due to a decrease in the propagation velocity. 2) The individual motor units may become to fire synchronously. 3) Larger motor units are recruited to replace the fatigued smaller motor units.

Despite many studies, the mechanism underlying muscular fatigue is still unclear. In this paper, the fatiguing process was studied on antagonistic muscles (jaw opener and closer).

\section{Methods}

The experiments were performed on six males with natural dentitions. Ages ranged from 21 to 33 years. No special effort was made to classify the subjects as normal or dysfunctional. However, no subject complained of symptoms of disturbances of the masticatory system. Bipolar recordings were made from the right masseter and anterior digastric muscles using single-unit surface electrode pair ${ }^{6)}$. The electrode was set parallel to the muscle fiber.

The subject was asked to sustain maximum isometric contractions of the jaw closing muscle in intercuspal position (centric occlusion) or on the jaw opening muscle against the thumb placed under the chin as long as possible. During the fatiguing experiment two EMG recordings were stored on magnetic tape (Sony: A-69, $9.5 \mathrm{~cm} / \mathrm{s}$ ). Off-line records were digitized at a sampling rate of $1 \mathrm{msec}$ and a 9 bits resolution. Then, the power spectral density function (PSDF), cross spectrum and coherence spectrum were calculated using fast Fourier transform (FFT) algorithm. The FFT was performed on 256 data points corresponding to $0.25 \mathrm{sec}$ of data. Mean PSDF was obtained by averaging five successive PSDFs. For these procedures, a data processor (Nihon Kohden: ATAC-450) was employed. The results were printed out on a digital plotter after the treatment processes (five points moving average and normalization). 


\section{Results and Discussion}

Maximum isometric contraction of the jaw closing muscle (clenching) in intercuspal position caused muscle fatigue. After the exercise, all of the subjects reported pain or soreness in the cheek or temple. The clenching time ranged from 23 to $61 \mathrm{sec}$ (mean $42.8 \mathrm{sec}$ ). During clenching the amplitude of the masseter muscle activity tended to decrease with fatigue. The power spectra obtained from the masseter muscle during the exercise were shifted to lower frequencies (Fig. 1). Also, the bandwidth became narrower than those obtained at the beginning of this exercise. These results agree with those of the previous studies ${ }^{2-5}$.

As reported by Kawamura et al. (1956) ${ }^{1)}$ the antagonistic muscle (anterior digastric muscle) was also active during the clenching effort. Although the intensity of antagonistic activity largely varied between the subjects, muscle activity tended to be more active at the end of the exercise.

Several possible explanations have been proposed for the EMG power spectrum shift. Palla and Ash (1981) $)^{4)}$ and Mills (1982) ${ }^{\text {r) }}$ suggested that the shift can be largely accounted for by a decrease in the conduction velocity of the action potential along the muscle fiber. Naeije and Zorn $(1982)^{8)}$, however, have measured the actual muscle fiber action potential conduction velocity

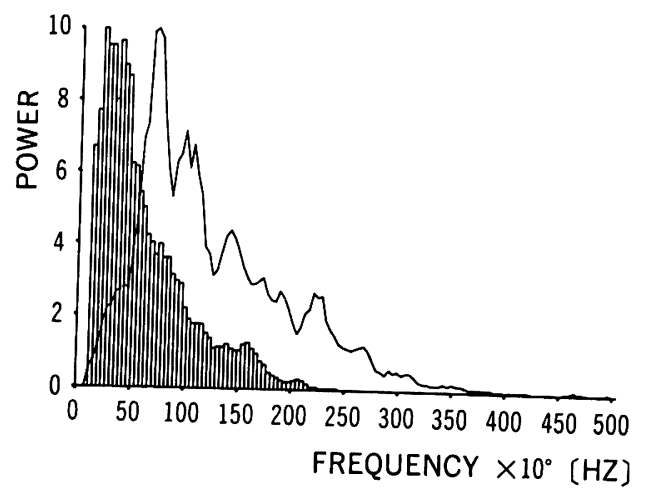

Fig. 1 Power spectra of the right masseter muscle at beginning of clench (broken line) and during fatigue (bar) during fatigue as well as the power spectrum shift in the human $\mathrm{m}$. biceps brachii, and found that an EMG power spectrum shift may occur during fatigue without a concomitant change in conduction velocity. Thus, mechanisms in the spectrum shift other than the change in conduction velocity were tested.

Frequently, the anterior digastric muscle was active during clenching. Therefore, the possibility of change in the firing frequency of motor units through synchronization of firing was studied on these antagonistic muscle activities observed at the end of the clenching effort. Since the masseteric motoneurons and the anterior digastric motoneurons are under reciprocal control ${ }^{9)}$, if synchronization is one of the main factors, then there may be certain synchronized peaks in the spectra of the antagonistic muscle activities. Thus, the cross spectrum and coherence spectrum were obtained from the right masseter and anterior digastric muscles. There was, however, no evidence to suggest the reciprocal and/or synchronized firing of the muscles in these frequency analyses (Fig. 2).

In order to test the last mechanism, fatigue of the digastric muscle was investigated. The maximum isometric contraction of the digastric muscle was sustained by supporting the jaw by hand. As in the jaw closer, the exercise also caused muscle fatigue in the jaw opener with pain or soreness. However, the power spectrum obtained from the anterior digastric muscle during isometric contraction remained identical to those observed at the beginning of this exercise (Fig. 3).

Histochemical studies on muscle fiber composition have been carried out on human masticatory muscles. According to these studies, masseter ${ }^{10)}$, temporal ${ }^{11)}$, and anterior digastric $^{12)}$ muscles contain $42 \%, 67 \%$, and $45.8 \%$ of fatigue sensitive fibers, respectively. If the power spectrum shift is mainly due to change in the activated muscle fibers, there would be more pronounced spectrum shift in the anterior digastric muscle than in the temporal muscle ${ }^{4}$. However, no shift was observed except for a slight decrease of the bandwidth in the jaw opening muscle 

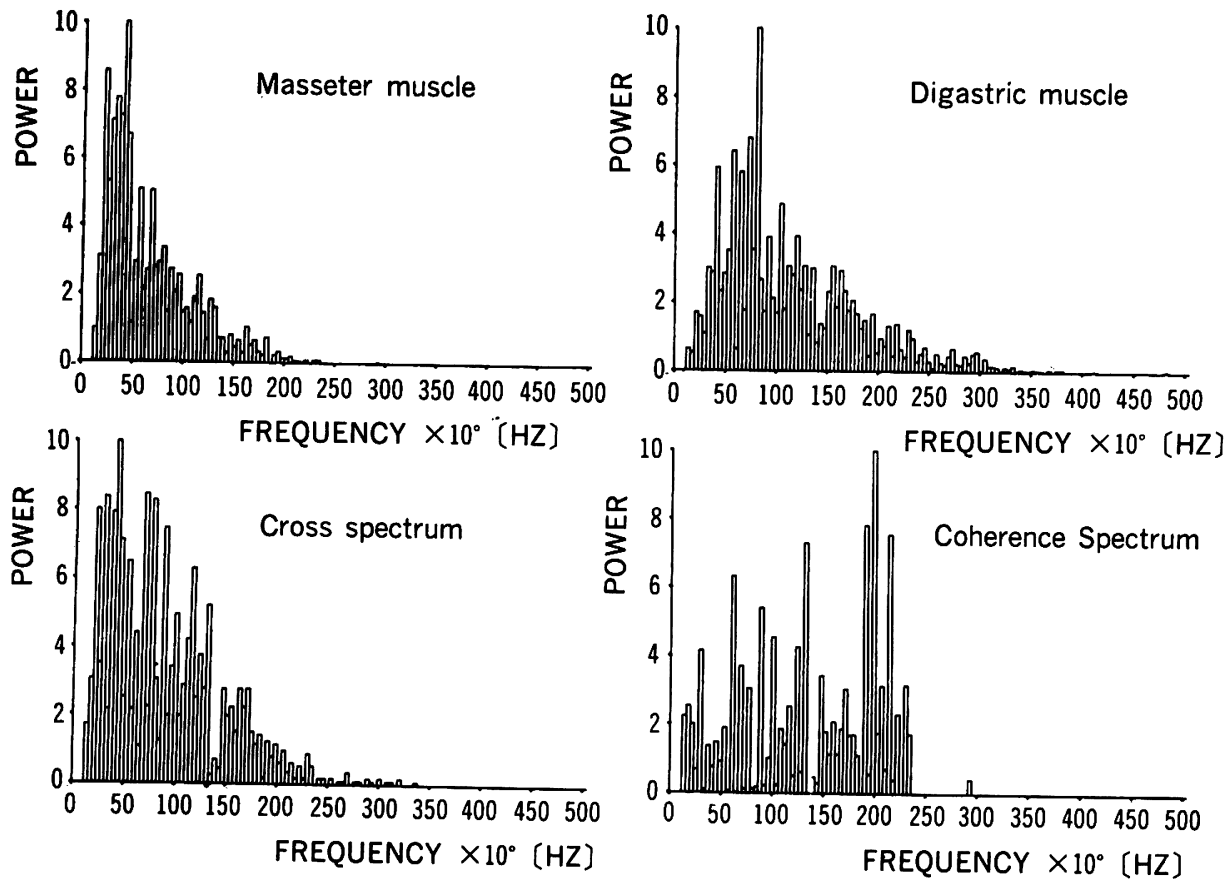

Fig. 2 Auto, cross, and coherence spectra of the right masseter and anterior digastric muscles during fatigue

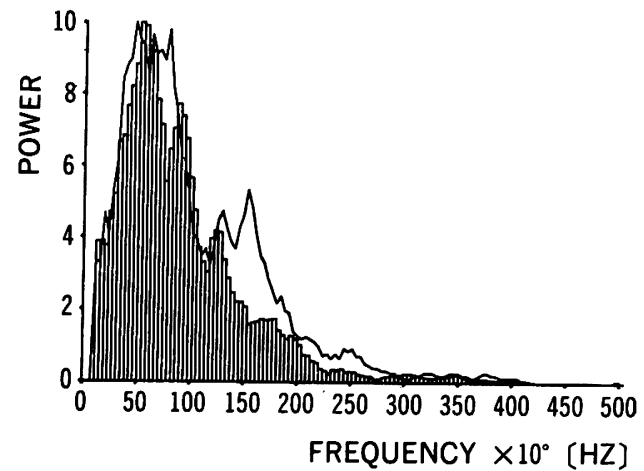

Fig. 3 Power spectra of the right anterior digastric muscle at the beginning of active jaw opening (broken line) and during fatigue (bar)

activity during fatigue. This unexpected result may be explained by changes in the metabolic state of the muscle. As suggested by many studies ${ }^{4,6,7)}$, forceful contraction may result in an increase of metabolic byproducts caused by blood-flow reduction. In the case of the digastric muscle, the bloodflow reduction might be small enough to cause such metabolic change, because the size of the muscle is small and its contractility is small when compared with the jaw closing muscles.

It is concluded that the power spectrum shift observed during isometric contraction is mainly due to a slowing of the conduction velocity of the action potential along the muscle fiber due to its metabolic changes.

\section{References}

1) Kawamura, Y., Fujimoto, J., Funakoshi, M. and Miki, K.: Studies on the effects of continuous forced biting. J. Osaka Univ. Dent. Sch. 1: 47-58, 1956.

2) Christensen, L. V.: Influence of muscle pain tolerance on muscle pain threshold in experimental tooth clenching in man. J. Oral Rehab. 6: 211-217, 1979.

3) Naeije, M. and Zorn, H.: Changes in the power spectrum of the surface electromyogram of the human masseter muscle due to local musclar fatigue. Archs. Oral Biol. 26: 409412, 1981.

4) Palla, S. and Ash, M. M. Jr.: Power spectral analysis of the surface electromyogram of 
human jaw muscles during fatigue. Archs. Oral Biol. 26: 547-553, 1981.

5) Lindström, L. and Hellsing, G.: Masseter muscle fatigue in man objectively quantified by analysis of myoelectric signals. Archs. Oral Biol. 28: 297-301, 1983.

6) Yamaga, T., Yamada, Y. and Ishioka, K. : Single-unit surface electrode pair for use in electromyography. J. Prost. Dent. 48: 626, 1982.

7) Mills, K. R.: Power spectral analysis of electromyogram and compound muscle action potential during muscle fatigue and recovery. J. Physiol. 326: 401-409, 1982.

8) Naeije, M. and Zorn, H.: Relation between EMG power spectrum shifts and muscle fibre action potential conduction velocity changes during local muscular fatigue in man. Eur.
J. Appl. Physiol. 50: 23-33, 1982.

9) Nakamura, Y., Takatori, M., Nozaki, S. and Kikuchi, M.: Monosynaptic reciprocal control of trigeminal motoneurons from the medial bulbar reticular formation. Brain Res. 89: 144-148, 1975.

10) Ringqvist, M.: Fiber sizes of human masseter muscle in relation to bite force. J. Neurol. Sci. 19: 297-305, 1973.

11) Ringqvist, M.: A histochemical study of temporal muscle fibers in denture wearers and subjects with natural dentition. Scand. J. Dent. Res. 82: 28-39, 1974.

12) Eriksson, P.-O., Eriksson, A., Ringqvist, M. and Thornell, L.-E.: Histochemical fibre composition of the human digastric muscle. Archs. Oral Biol. 27: 207-215, 1981. 